\title{
VEGETATION COVER ANALYSIS IN SHAANXI PROVINCE OF CHINA BASED ON GRID PIXEL TERND ANALYSIS AND STABILITY EVALUATION
}

\author{
H. Yue ${ }^{1}$, Y. Liu $^{1 *}$ \\ ${ }^{1}$ College of Geomatics, Xi'an University of Science and Technology, Xi'an, Shaanxi 710054, China - yue_hui@live.com, \\ liuying712100@163.com
}

Commission III, WG III/7

KEY WORDS: Vegetation, MODIS, NDVI, Trend analysis, Stability evaluation, Shaanxi

\begin{abstract}
:
As a key factor affecting the biogeochemical cycle of human existence, terrestrial vegetation is vulnerable to natural environment and human activities, with obvious temporal and spatial characteristics. The change of vegetation cover will affect the ecological balance and environmental quality to a great extent. Therefore, the research on the causes and influencing factors of vegetation cover has become the focus of attention of scholars at home and abroad. In the evolution of human activities and natural environment, the vegetation coverage in Shaanxi has changed accordingly. Using MODIS/NDVI 2000-2014 time series data, using the method of raster pixel trend analysis, stability evaluation, rescaled range analysis and correlation analysis, the climatic factors in Shaanxi province were studied in the near 15 years vegetation spatial and temporal variation and influence of vegetation NDVI changes. The results show that NDVI in Shaanxi province in the near 15 years increased by 0.081, the increase of NDVI in Northern Shaanxi was obvious, and negative growth was found in some areas of Guanzhong, southern Shaanxi NDVI overall still maintained at a high level; the trend of vegetation change in Shaanxi province has obvious spatial differences, most of the province is a slight tendency to improve vegetation, there are many obvious improvement areas in Northern Shaanxi Province. Guanzhong area vegetation area decreased, the small range of variation of vegetation in Shaanxi province; the most stable areas are mainly concentrated in the southern, southern Yanan, Yulin, Xi'an area of Weinan changed greatly; Shaanxi Province in recent 15a, the temperature and precipitation have shown an increasing trend, and the vegetation NDVI is more closely related to the average annual rainfall, with increase of $0.48^{\circ} \mathrm{C} / 10$ years and $69.5 \mathrm{~mm}$ per year.
\end{abstract}

\section{INTRODUCTION}

Vegetation is a community composed of plants, such as forests, grasslands, shrubs and meadows, which is distributed on the land surface. It is the most important part of terrestrial ecosystems. As a key factor affecting the biogeochemical cycle of human existence, terrestrial vegetation is vulnerable to natural environment and human activities, with obvious temporal and spatial characteristics. Changes in vegetation cover will affect the local ecological balance and environmental quality. Research on the dynamic change of vegetation has become a focus of domestic and foreign scholars. Remote sensing as an important vegetation research technology has developed rapidly in recent years (Zhang et al., 2017), mainly in the identification of plants and land cover types (Song et al., 2006), crops estimated production (Xu et al., 2009), macrovegetation monitoring (Ma et al., 2017), biomass estimation and inversion of biophysical parameters (Fang and Zhang, 2003; Qin et al., 2015). The normalized difference vegetation index (NDVI) obtained from satellite remote sensing can indicate the change characteristics of vegetation coverage and greenness. NDVI has been widely used in monitoring vegetation activity at large scale. To analyse the spatiotemporal variation of vegetation cover in Shaanxi province is not deep enough, therefore, this paper uses MODIS NDVI 2000-2014 data, prediction analysis method, pixel grid stability evaluation and trend based on the temporal and spatial characteristics of vegetation in Shaanxi Province, and explore the reasons for the change of May, in order to enrich the academic knowledge about Shaanxi vegetation to climate change and human activities response characteristics, and the study can also provide direction and guidance for environmental protection and governance, can also provide reference for the coordination of economic development and environmental protection.

Shaanxi province is located in the northwest of China. It is located between 105 degree 29 'to 111 degree 15' and 31 degrees 42 'to 39 degrees' 35 ' in the north latitude of East China, with an area of 205 thousand and $800 \mathrm{~km}$. Shaanxi Province is located inland in the northwest of China and in the middle of the Loess Plateau in the north. Soil erosion, sandification and other environmental problems are severe in recent years. In the evolution of human activities and the natural environment, the vegetation coverage in Shaanxi Province has also changed. At present, research on vegetation change in Shaanxi Province has achieved certain results (Li et al., 2015; Liu et al., 2017; Zhang and Yan, 2017). On the whole, related studies have studied the temporal and spatial distribution characteristics and influencing factors of vegetation changes in Shaanxi Province, but few studies have been conducted on the future changes in vegetation in this region.

\section{DATA AND METHODS}

\subsection{Data}

Utilizing the MODIS Vegetation Index Product (MOD13A3) of Shaanxi Province with spatial resolution of $1 \mathrm{~km}$ provided by

* Corresponding author 
the US Earth Resources Observation Science Center from 2000 to 2014 .

\subsection{Grid pixel trend analysis}

Grid pixel trend analysis can simulate the changing trend of each raster cell. By this method, we can simulate the trend of NDVI and analyze the change of vegetation coverage.

$$
\text { slope }=\frac{n \times \sum_{i=1}^{n}\left(t \times N D V t_{t}\right)-\sum_{i=1}^{n} t \times \sum_{i=1}^{n} N D V I_{t}}{n \times \sum_{i=1}^{n} t_{t}^{2}-\left(\sum_{i=1}^{n} t_{t}\right)^{2}}
$$

In the formula, the variable $\mathrm{I}$ is synthesis image arranged by time sequence; $\mathrm{n}$ is the number of samples; $\mathrm{NDVI}_{\mathrm{i}}$ for the I image NDVI value; $T_{i}$ is the I image corresponding to the time, 2000 - 2014 NDVI slope is a linear regression slope, if slope $>0$, the period of NDVI rise the vegetation cover has been improved, whereas NDVI decreased, the deterioration of the vegetation cover.

\subsection{Stability evaluation}

The fluctuation value indicates that the vegetation community is greatly disturbed and unstable, and the state of vegetation community in the fluctuation value novel is stable. The variation coefficient of NDVI from 2000 to 2014 is calculated by formula (2) and (3), so as to reflect the relative fluctuation of vegetation on different pixels in the study area in the past 15 years.

$$
\begin{aligned}
& S=\sqrt{\frac{\sum_{i=1}\left(N D V L_{i}-\overline{N D F}\right)^{2}}{n-1}} \\
& C_{V}=\frac{s}{W D 2}
\end{aligned}
$$

\section{RESULTS AND ANALYSIS}

\subsection{Analysis of the change trend of NDVI in Shaanxi Province}

In the whole province, the slope of the change trend line of NDVI in Shaanxi province is between -0.1-0.1, parts of the western and south-western of Shaanxi Province remained unchanged or slightly degraded. The eastern and central parts of Shaanxi improved significantly, while the rest areas were slightly improved. The unchanged vegetation in the whole province accounted for $52 \%$, slight improvement accounts for $42.8 \%$, slight degradation accounts for $3.5 \%$, moderate improvement accounts for $1.46 \%$, moderate degradation accounts for $0.15 \%$, and serious degradation and obvious improvement accounts for no more than $0.01 \%$. It shows that the vegetation coverage in Shaanxi is improved. Most of the Shaanxi's vegetation showed a slight improvement trend, and the area of vegetation in northern Shaanxi significantly improved, mainly due to the implementation of the national policy of returning farmland to forests, "Three North" protection forest engineering grass, ecological restoration and reconstruction, and prevention and control of desertification. The population in the Guanzhong area is concentrated, and the type of vegetation is mostly crops, which are easily affected by human activities. With the increase in the number of people, the urbanization construction has reduced the area covered by vegetation. In the mountainous areas in southern Shaanxi, the vegetation is rich in species, large in quantity, complex in topography and landform, and affected little by human activities, resulting in a small range of change, mainly in urban concentrated areas.

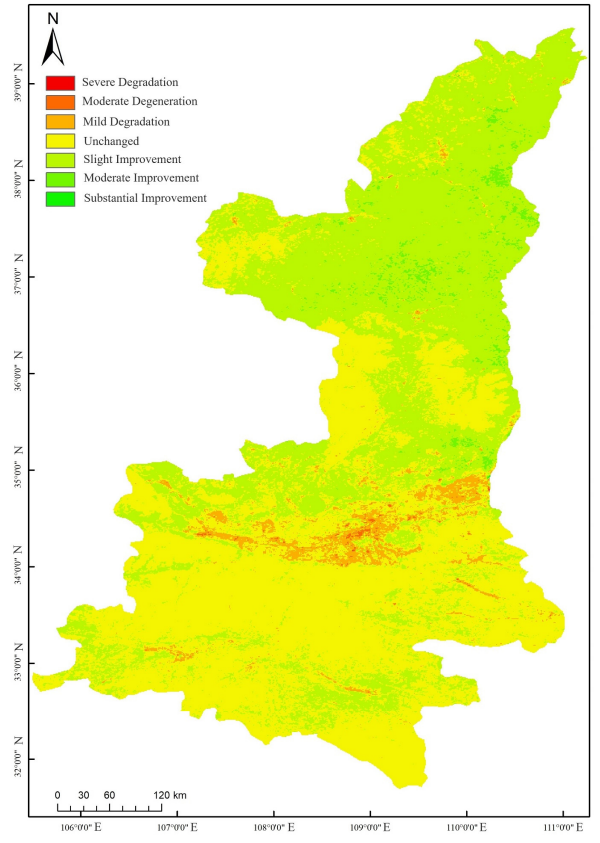

(a)

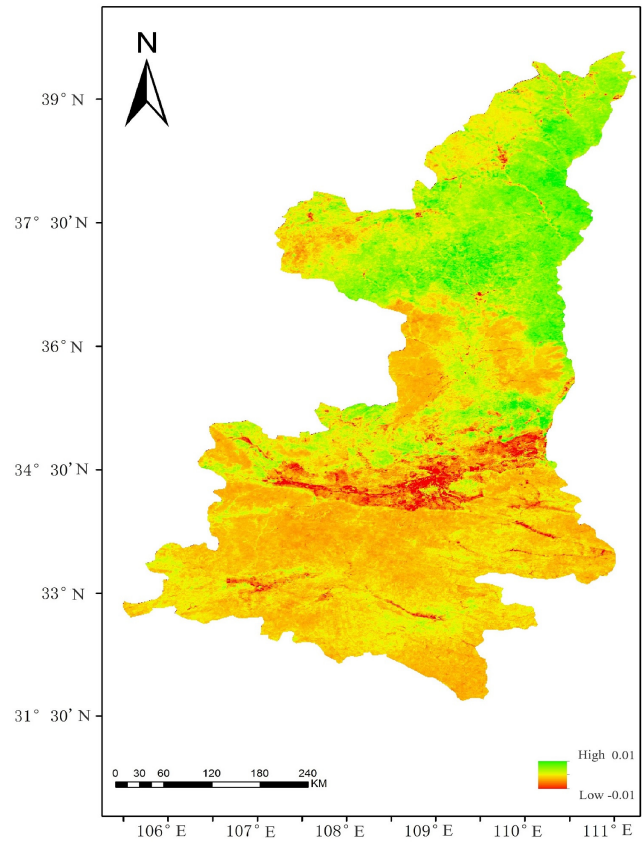

(b)

Figure 1. NDVI trend map of Shaanxi Province (a) Slope of NDVI change (b) NDVI trend level

\subsection{Stability analysis of NDVI in Shaanxi Province}

The range of variation coefficient is between $0.004-4.475$, the most stable vegetation areas are mainly concentrated in the southern of Shaanxi and southern Yan'an, the coefficient of variation between 0-0.1; Xi'an, part of central Xianyang, northern Weinan, northern Yan'an and Yulin change medium, coefficient of variation between 0.1-0.2, a small area of northern Yulin changed greatly, the coefficient of variation was above 1. More than half of the vegetation was stable in the 
province, $28 \%$ of the middle degree, and less than $2 \%$ of the instability. It shows that the vegetation of Shaanxi province is



(a) relatively stable and the degree of change is small in Shaanxi province.

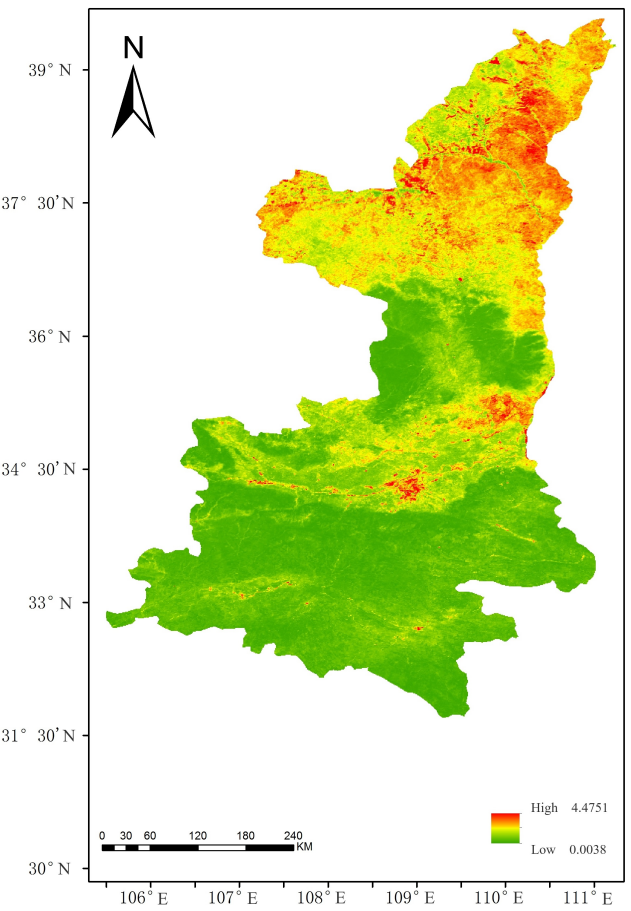

(b)

Figure 2. Stability analysis diagram of Shaanxi NDVI (a) NDVI coefficient of variation（b ) NDVI stability level

\section{RESULTS AND DISCUSSION}

During the past 15 years, the average NDVI value of vegetation in Shaanxi increased from 0.4273 in 2000 to 0.4942 in 2015, and the NDVI increased 0.067 in the total 15 years, with an increase speed of $16.0 \%$. The Northern NDVI increased significantly in northern Shaanxi, but overall it remained low. The growth of NDVI in the Guanzhong area was relatively slow, with negative growth in some areas. The area of high NDVI in southern Shaanxi increased significantly and remained at a relatively high level. The trend of vegetation change in Shaanxi Province has obvious spatial differences. Most of Shaanxi's vegetation showed a slight improvement trend. The area of significant improvement in northern Shaanxi was larger, and the area covered by vegetation in Guanzhong was reduced, while the change in southern Shaanxi was small. During the 15a period, the most stable vegetation areas in Shaanxi Province were mainly concentrated in southern Shaanxi, southern Yan'an, parts of Xi'an, central Xianyang, northern Weinan, northern Yan'an, and most parts of Yulin. The changes ranged moderately in Yulin, Xi'an, and Weinan. In order to prevent environmental degradation and degradation of vegetation in northern Shaanxi, we should continue to vigorously implement the national policy of returning farmland to forests, "Three North" protection forest engineering grass, ecological restoration and reconstruction, and prevention and control of desertification; the expansion of construction land in the Guanzhong region should be carried out in an orderly manner.

\section{ACKNOWLEDGEMENTS (OPTIONAL)}

This work is partially supported by grants from The National Natural Science Foundation of China (Grant No. 41401496).

\section{REFERENCES}

Fang Xiuqing, Zhang Wanchang., 2003. The application of remotely sensed data to the estimation of the leaf area index. Remote Sensing for Land and Recourses, 15(3), pp.58-62.

Li Dengke, Fan Jianzhong, Quan Wenting., 2015. Analysis of vegetation degradation and its driving factors in Shaanxi Province. Chinese Journal of Ecology, 34(10), pp.2907-2913.

Liu Ying, Hou Enke, Yue Hui., 2017. Remote Sensing Monitoring of Vegetation Changes in Shaanxi Based on MODIS. Yellow River, 39(3), pp.90-94.

Ma Lei, Yan Haowen, He Yi, et al., 2017. Vegetation changes in south Himalayas areas based on remote sensing monitoring during 2001-2015. Arid Land Geography, (2), pp. 405-414.

Qin Chao, Li Junyi, Chen Hongfei, et al., 2015. Temporal and spatial evolution of vegetation cover and its relationship with climate factors in Shaanxi Province. Chinese Journal of Agrometeorology, 36(1), pp.108-114.

Song Kaishan, Zhang Bai, Wang Zongming, et al., 2006. Inverse model for estimating soybean chlorophyll concentration using in-situ collected canopy hyperspectral data. Transactions of the Chinese Society of Agricultural Engineering, 22(8), pp.16-21.

Xu Xingang, Wang Jihua, Huang Wenjiang, et al., 2009. Estimation of crop yield based on weight optimization combination and multi-temporal remote sensing data. Transactions of the Chinese Society of Agricultural Engineering, 25(9), pp.137-142. 
Zhang Hua, Zhang Gaigai, Wu Rui., 2017. Object-based vegetable classification based on GF-1 imagery in Minqin Oasis. Arid Land Geography, 40(4), pp.831-83.

Zhang Jun, Yan Junping., 2017. Characteristics of NDVI changes under the different vegetation types in Shaanxi Province from 1982 to 2013. Journal of Arid Land Resources and Environment, 31(4), pp.86-92. 